\title{
A Combined Time and Frequency Domain Approach to Channel Estimation for SFBC MIMO-OFDM Wireless Communication System
}

\author{
Gajanan R Patil \\ Army Institute of Technology \\ Dighi Hills, Alandi Road \\ Pune-411015
}

\author{
Vishwanath K Kokate \\ Sinhgad College of Engg. \\ Vadgaon Bk. \\ Pune-411041
}

\begin{abstract}
This paper presents a combined approach to channel estimation for multiple input multiple output (MIMO) orthogonal frequency division multiplexing (OFDM). It uses both time domain and frequency domain information in the received signal to estimate the channel. Initial estimate of the channel is obtained using pilot assisted least square (LS) channel estimation. The estimate is further enhanced by extracting information through the received data symbols. A frequency domain approach is used to estimate the channel using pilots whereas time domain approach is used to enhance the estimate of the channel. The performance of the proposed estimator is studied under various channel models. The simulation study shows that this approach outperforms the pilot assisted least square channel estimation method.
\end{abstract}

\section{General Terms}

Channel Estimation, MIMO-OFDM, Wireless Communication.

\section{Keywords}

Multiple input multiple output, orthogonal frequency division multiplexing, pilot assisted least square channel estimation, space frequency block coding.

\section{INTRODUCTION}

The demand of high data rates for wireless communication can be catered by use of multiple antennas both at transmitter and receiver end. The use of simple transmit diversity technique and Space time block codes for a MIMO system was proposed initially by Alamouti [1] and V Tarokh et. al [2], [3]. Orthogonal frequency division multiplexing converts frequency selective fading channel into set of frequency flat channels thereby combating the effect of fast fading. Multiple-input multiple-output (MIMO) wireless technology in combination with orthogonal frequency division multiplexing (OFDM) is an attractive air-interface solution for next-generation wireless local area networks (WLANs), wireless metropolitan area networks (WMANs), and fourthgeneration mobile cellular wireless systems. OFDM directly extends to MIMO channels with IFFT/FFT and CP operations being performed at each transmit and receive antennas [4]-[6]. MIMO signaling for Single Carrier (SC) modulation in frequency-flat fading channels can be overlaid easily on OFDM by simply performing operations on a tone-by-tone basis [7]. Both spatial diversity and spatial multiplexing MIMO OFDM systems can be implemented. Space frequency coded MIMO-OFDM discussed in [8] consists of space time block coded symbols occupying adjacent tones in the same
OFDM symbol. The bit stream to be transmitted is first modulated then encoded and interleaved. The resulting data symbols to be transmitted are mapped across space and frequency by a space-frequency encoder such as the one described in [9], [10]. The receiver demodulates the received signal and estimates the transmitted space-frequency codeword followed by de-interleaving and decoding. A space time frequency approach for MIMO-OFDM system is discussed in [11].

The system's ability to achieve MIMO capacity depends on channel state information. Accurately estimating MIMO channel is much more challenging than SISO channel [12] [13]. There are number of channel estimation schemes suggested in literature. These schemes can be categorized as training based channel estimation (TBCE), blind channel estimation (BCE) and semi-blind channel estimation (SBCE). Training based schemes are capable of accurately estimating a MIMO channel, provided a large training overhead is made available. Hence there is considerable reduction in system throughput [14]. Blind methods do not require the training overhead. However these methods not only impose high complexity and slow convergence, but also suffer from unavoidable estimation and decision ambiguities [15]-[17]. Semi-blind methods offer attractive practical means of implementing MIMO systems. Semi-blind channel estimation schemes, use a few training symbols to provide the initial MIMO channel estimation and make use of blind information to further improve the estimation [16] [18] [19].

The training based channel estimation in MIMO-OFDM system can be block type or comb type. In block type method the pilots are inserted into all tones of a OFDM symbol so that the channel estimate of each tone can be obtained in frequency domain. This type of technique is suitable when the channel is varying slowly. Comb type method inserts pilots in the tones at fixed interval in one OFDM symbol. The channel estimate for these tones is obtained using least squared (LS) or minimum mean squared error (MMSE) technique. The estimate of remaining tones is obtained by interpolation. The LS channel estimation with block-type pilot for MIMOOFDM was first proposed in [20], and was simplified in [21]. In MIMO-OFDM system, the received OFDM symbols can be processed in time domain or frequency domain. Number of semi-blind channel estimation methods for MIMO-OFDM system are proposed in literature. See for example [22] and references therein. Performance of Space frequency block coded MIMO-OFDM system is given in [23].

This paper presents a channel estimation method using both frequency and time domain approach for a MIMO-OFDM system. The initial estimate of the channel is obtained using 
comb-type pilot assisted LS estimation and interpolation. This estimate is further enhanced by using detected data symbols. The new approach clearly outperforms the simple training based approach. Rest of the paper is organized as follows. Section 2 describes the system model. The design of the proposed estimator is given in section 3. The simulation results and discussion on the results is given in section 4 . Finally section 5 gives the conclusion.

Throughout our discussions we adopt the following notational conventions. Boldface capitals and lower-case letters stand for matrices and vectors, respectively. I denote the identity matrix. $(.)^{T}(.)^{H}$ and $(.)^{\dagger}$ are transpose, conjugate transpose and Moore-Penrose pseudo inverse operators respectively.

\section{SYSTEM MODEL}

Consider a MIMO-OFDM system with $N_{T}$ transmit and $N_{R}\left(>N_{T}\right)$ receive antennas. Let there be $K$ subcarriers in one OFDM symbol. It is assumed that time-variant wireless channel obey Rayleigh distribution and is quasi-static in one OFDM block duration. The maximum multipath delay length is $L$. The length of Cyclic Prefix (CP) is chosen to be longer than $L$. Channels between each transmit and receive antenna pairs are assumed to be mutually uncorrelated. The channel impulse response between $j$-th receive and $i$-th transmit antenna corresponding to $l$-th path delay is denoted as $\mathrm{h}_{i j}(l)$; where $l=0,1,2 \ldots L-1$.

At a transmission time $\mathrm{n}$, binary data is grouped according to type of modulation (MQAM or MPSK) and mapped onto different sub-carriers depending on the coding to be used. Then the signal on $k$ th sub-carrier at ith transmit antenna is denoted by $\mathrm{X}_{\mathrm{i}}[\mathrm{n}, \mathrm{k}]$ where $i=1,2, \ldots, N_{T}, k=0,1,2, \ldots, K$ $-1, n=0,1,2, \ldots, N-1$. The received signal in time domain and frequency domain at $j$-th receive antenna is given by (1) and (2) respectively [21], [24].

$\mathbf{y}_{\mathrm{j}}(\mathrm{n})=\sum_{\mathrm{i}=1}^{\mathrm{N}_{\mathrm{T}}} \mathbf{h}_{i j}(\mathrm{n}) \otimes \mathbf{x}_{\boldsymbol{i}}(\mathrm{n})+\mathbf{w}_{\boldsymbol{j}}(\mathrm{n})$

$Y_{j}[n, k]=\sum_{i=1}^{N_{T}} H_{i j}[n, k] X_{i}[n, k]+W_{j}[n, k]$

Where $j=1,2, \ldots, N_{R}, \mathrm{H}_{\mathrm{ij}}[\mathrm{n}, \mathrm{k}]$ is the frequency response between antennas $i$ and $j, \mathrm{~W}_{\mathrm{j}}[\mathrm{n}, \mathrm{k}]$ is the additive Gaussian noise with zero mean and variance $\sigma_{n}^{2}$. If $\boldsymbol{F}$ denotes DFT matrix then we have following relationships.

$$
\begin{aligned}
& \mathbf{X}_{i}[\mathrm{n}]=\mathrm{Fx}_{\boldsymbol{i}}(\mathrm{n}), \mathrm{Y}_{j}[\mathrm{n}]=\boldsymbol{F} \mathbf{y}_{j}(\mathrm{n}), \quad \mathbf{H}_{i j}[\mathrm{n}]=\boldsymbol{F} \mathbf{h}_{i j}(\mathrm{n}) \\
& \text { and } \mathbf{W}_{j}[\mathrm{n}]=\boldsymbol{F} \mathbf{w}_{j}(\mathrm{n})
\end{aligned}
$$

Now Let us represent the transmitted OFDM symbol from $i$ th antenna as,

$\mathbf{X}_{i}[\mathrm{n}]=\operatorname{diag}\left\{\mathrm{X}_{\mathrm{i}}[\mathrm{n}, 0], \mathrm{X}_{\mathrm{i}}[\mathrm{n}, 1] \ldots \mathrm{X}_{\mathrm{i}}[\mathrm{n}, \mathrm{K}-1]\right\} \in \mathbb{C}^{\mathrm{K} \times \mathrm{K}}$

The received OFDM symbol at $j$-th antenna as,

$\mathbf{Y}_{j}(\mathrm{n})=\left[\mathrm{Y}_{\mathrm{j}}[\mathrm{n}, 0], \mathrm{Y}_{\mathrm{j}}[\mathrm{n}, 1] \ldots \mathrm{Y}_{\mathrm{j}}[\mathrm{n}, \mathrm{K}-1]^{\mathrm{T}} \in \mathbb{C}^{\mathrm{K} \times 1}\right.$

The transmitted OFDM symbols from all transmit antennas as,

$\mathbf{X}(\mathrm{n})=\left[\mathrm{X}_{1}[\mathrm{n}] \mathrm{X}_{2}[\mathrm{n}] ; \ldots, \mathrm{X}_{N T}[\mathrm{n}]\right] \in \mathbb{C}^{\mathrm{K} \times \mathrm{KN}_{\mathrm{T}}}$

The channel gain on each subcarrier from $i$ th transmit and $j$ th receive antenna as,
$\mathbf{H}_{i j}[\mathrm{n}]=\left[\mathrm{H}_{i j}[\mathrm{n}, 0], \mathrm{H}_{i j}[\mathrm{n}, 1], \ldots \mathrm{H}_{i j}[\mathrm{n}, \mathrm{K}-1]\right]^{\mathrm{T}} \in \mathbb{C}^{\mathrm{K} \times 1}$

The entire channel gain matrix corresponding to all transmit and $j$ th receive antenna as,

$$
\mathbf{H}_{j}(\mathrm{n})=\left[\mathbf{H}_{1 j}^{T}[\mathrm{n}], \mathbf{H}_{2 j}^{T}[\mathrm{n}], \ldots, \mathbf{H}_{\boldsymbol{N}_{T}}^{T}[\mathrm{n}]\right]^{T} \in \mathbb{C}^{\mathrm{KN}_{\mathrm{T}} \times 1}
$$

The additive white Gaussian noise on each subcarrier at $j$-th receive antenna as,

$\mathbf{W}_{j}(\mathbf{n})=\left\{W_{\mathrm{j}}[\mathrm{n}, 0], W_{\mathrm{j}}[\mathrm{n}, 1] \ldots W_{\mathrm{j}}[\mathrm{n}, \mathrm{K}-1]\right\} \in \mathbb{C}^{\mathrm{K} \times 1}$

Using (4), (5), (7) and (8) we can express the received signal at $j$-th receive antenna in frequency domain as

$\mathbf{Y}_{\mathbf{j}}(\mathrm{n})=\mathbf{X}(\mathrm{n}) \mathbf{H}_{\mathbf{j}}(\mathrm{n})+\mathbf{W}_{\mathbf{j}}(\mathrm{n})$

Now the time domain representation of the channel between $i$ th transmit and $j$ th receive antenna is given by

$\mathbf{h}_{\mathrm{ij}}[\mathrm{n}]=\left[\mathrm{h}_{\mathrm{ij}}[\mathrm{n}, 0], \mathrm{h}_{\mathrm{ij}}[\mathrm{n}, 1], \ldots \mathrm{h}_{\mathrm{ij}}[\mathrm{n}, \mathrm{L}-1]\right]^{\mathrm{T}} \in \mathbb{C}^{\mathrm{L} \times 1}$

The time domain representation of the channel at $j$-th receive antenna from all transmit antennas is given by,

$\mathbf{h}_{\mathbf{j}}(\mathrm{n})=\left[\mathbf{h}_{\mathbf{1 j}}^{\mathrm{T}}[\mathrm{n}], \mathbf{h}_{2 \mathbf{j}}^{\mathrm{T}}[\mathrm{n}], \ldots, \mathbf{h}_{\mathbf{N}_{\mathrm{j}}}^{\mathrm{T}}[\mathrm{n}]\right]^{\mathrm{T}} \in \mathbb{C}^{\mathrm{LN} N_{\mathrm{T}} \times 1}$

The relationship between $H_{j}(n)$ and $h_{j}(n)$ is given by,

$\mathbf{H}_{\mathbf{j}}(\mathrm{n})=\boldsymbol{F}_{\boldsymbol{M}} \mathbf{h}_{\boldsymbol{j}}(\mathrm{n})$

Where, $\quad \boldsymbol{F}_{\boldsymbol{M}}=\operatorname{diag}[\boldsymbol{F} \times \mathbf{M}, \boldsymbol{F} \times \mathbf{M}, \ldots, \boldsymbol{F} \times \mathbf{M}] \in \mathbb{C}^{\mathrm{KN}_{\mathrm{T}} \times \mathrm{LN}_{\mathrm{T}}}$ and $\mathrm{M}=\left[\mathrm{I}_{\mathrm{L} \times \mathrm{L}} \mathrm{O}_{\mathrm{K}-\mathrm{L} \times \mathrm{L}}\right]^{T}$

Substituting (12) in (9) we get,

$\mathbf{Y}_{\mathbf{j}}(\mathrm{n})=\mathbf{X}(\mathrm{n}) \boldsymbol{F}_{\boldsymbol{M}} \mathbf{h}_{j}(\mathrm{n})+\mathbf{W}_{j}(\mathrm{n})$

Now let, $\quad \mathbf{A}=\mathbf{X}(\mathrm{n}) \boldsymbol{F}_{\boldsymbol{M}}$ hence (13) can be written as,

$\mathbf{Y}_{j}(\mathrm{n})=\mathbf{A} \mathbf{h}_{j}(\mathrm{n})+\mathbf{W}_{\boldsymbol{j}}(\mathrm{n})$

Equation (9) and (14) are frequency and time domain representations of the received signal at $j$-th antenna respectively during transmission time $n$. These expressions will be used in this paper for channel estimation at the receiver.

\subsection{Pilot placement}

There are main types of pilot placements in MIMO-OFDM systems. In a block-type pilot MIMO-OFDM system, the orthogonal pilots are assigned to all subcarriers in a OFDM symbol. These pilots are transmitted periodically. From these pilots, the channel is estimated and used for detection of data carried by subsequent OFDM symbols. Block type pilot placement is useful where the channel is time invariant over number of OFDM symbols i.e. channel is slow fading. In the Comb-type pilot MIMO-OFDM system, pilots are inserted into a set of subcarriers in a OFDM symbol and channel is estimated for rest of the subcarriers using interpolation. This type of pilot placement is useful where the channel is fast fading. The block-type and comb-type pilot placement is shown in fig 1. 


\subsection{SFBC MIMO-OFDM}

In SFBC MIMO-OFDM system, the input data symbols $S_{0}, S_{1} \ldots S_{K-1}$ are converted into $K$ parallel symbols. These symbols are divided into $K / N_{T}$ groups. Each of these groups is coded using space time block coding and transmitted on

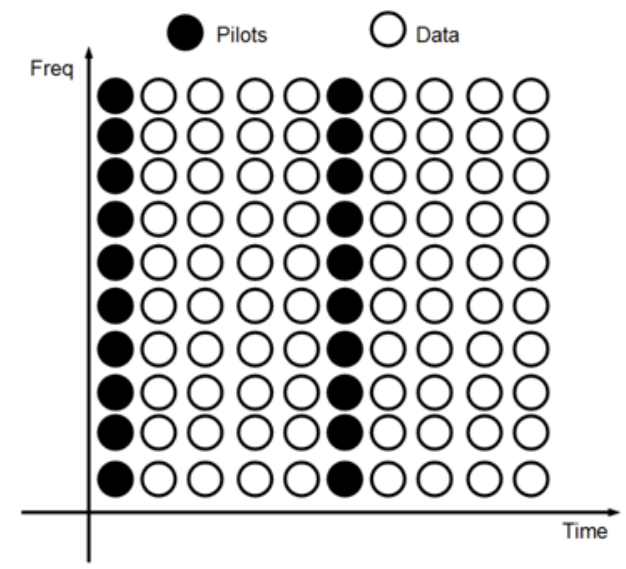

(a) Block Type different subcarriers on $N_{T}$ different antennas. For example, for $N_{T}=2$ G2 coded symbols are transmitted on two antennas as below

$$
\boldsymbol{X}=\left[\begin{array}{ccccccc}
S_{0} & -S_{1}^{*} & S_{2} & -S_{3}^{*} & & S_{K-2} & -S_{K-1}^{*} \\
S_{1} & S_{0}^{*} & S_{3} & S_{2}^{*} & \cdots & S_{K-1} & S_{K-2}^{*}
\end{array}\right]
$$

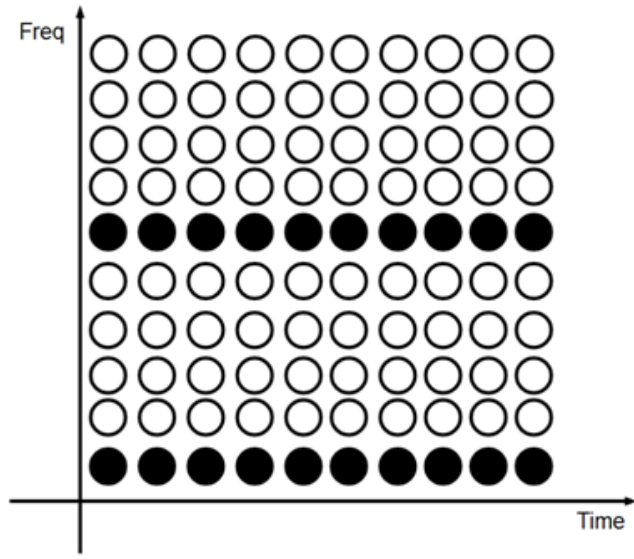

(b) Comb Type

Fig. 1: Pilot and data arrangement on each transmit antenna

\section{ESTIMATOR DESIGN}

The channel estimation in SFBC MIMO-OFDM system can be obtained by frequency domain or time domain processing. Let us use the block-type pilot arrangement in which out of $K$ subcarriers, $N p$ orthogonal pilots are periodically placed. Let these set of pilots be denoted as $\boldsymbol{X}_{\boldsymbol{p}}$. Then the frequency domain channel estimation is given by (16) which is obtained applying least square (LS) estimation to (9).

$\widehat{\boldsymbol{H}}_{j}[n, k]=\boldsymbol{X}_{\boldsymbol{P}}^{\dagger}\left[n, k: k+N_{T}\right] \boldsymbol{Y}_{j}[n, k]$

where $\quad k=0, \frac{K}{N p}, \frac{2 K}{N p}, \ldots . \frac{(N p-1) K}{N p}$.

Channel gains of remaining subcarriers are obtained using interpolation. Using the channel estimate of each subcarrier, the data sent on the corresponding subcarriers is detected using space frequency block decoding technique [25]. The detected symbols are used to further enhance the channel estimate. Time domain channel estimation is used for this purpose. If the detected symbols are arranged again as (15), the time domain channel estimation is given by (17) which is obtained by applying LS estimation to (14).

$\widehat{\boldsymbol{h}_{\boldsymbol{J}}}=\boldsymbol{A}^{\dagger} \boldsymbol{Y}_{j}$

Where, $\boldsymbol{A}=\boldsymbol{X} \boldsymbol{F}_{\boldsymbol{M}}$.

The frequency domain estimate is then obtained as

$\widehat{\boldsymbol{H}}_{j}=\boldsymbol{F} \widehat{\boldsymbol{h}}_{\boldsymbol{j}}$

Using this enhanced estimate of the channel, the data is detected which will be more accurate than the pilot assisted LS estimator. Hence the BER performance of the proposed scheme is better than the pilot assisted LS estimator. This advantage is achieved at increased computational complexity.

\section{SIMULATION RESULTS AND DISCUSSION}

A SFBC MIMO-OFDM system with 2 transmit and 4 receive antennas was simulated. Alamouti's [1] code is used for performance evaluation. Table I gives the specifications of the system which are similar to IEEE 802.16a Broadband Wireless Access [26]

Table 1. Simulation parameters for MIMO-OFDM System

\begin{tabular}{|l|l|}
\hline System Parameters & Parameter Value \\
\hline Modulation & QPSK, 64 QAM \\
\hline Channel Bandwidth & $20 \mathrm{MHz}$ \\
\hline FFT/IFFT size & 256 \\
\hline Cyclic Prefix Length & $64(25 \%)$ \\
\hline Pilots placement & Comb-type \\
\hline
\end{tabular}

The channel models used in the simulation are modified Stanford University Interim (SUI) channel models described in [27]. The channel is assumed to be quasi static i.e. it remains constant for one OFDM symbol duration. In the simulation, the modulation scheme is same for all data and pilot subcarriers with average energy $E_{s}$ and the noise is complex additive white Gaussian with zero mean and variance $N_{o} / 2$.

From the results in fig. 2 we observe that the proposed scheme has an advantage of almost $2 \mathrm{~dB}$ over the pilot assisted LS channel estimation. The performances of proposed frequency and time domain combined scheme result into steady decay rate for low delay spread channel models SUI-1, SUI-2 and SUI-3. The rest of the models i.e. SUI-4, SUI-5 and SUI-6 exhibit low decay rate at high SNR as they have large delay spread. Table 2 gives some tabulated values of BER with 
$\mathrm{Eb} / \mathrm{No}=6 \mathrm{~dB}$ for $\mathrm{LS}$ and proposed estimator

In fig. 3 we present the BER performance with SUI-1 and SUI-5 channel models for a similar setup as described above except that the modulation scheme used is QPSK

From the results of fig. 3 we find that the BER performance of

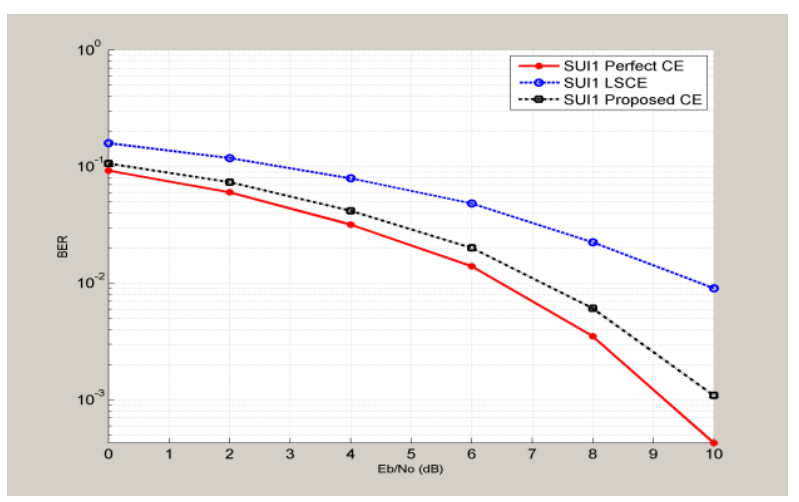

(a) SUI-1 Channel Model

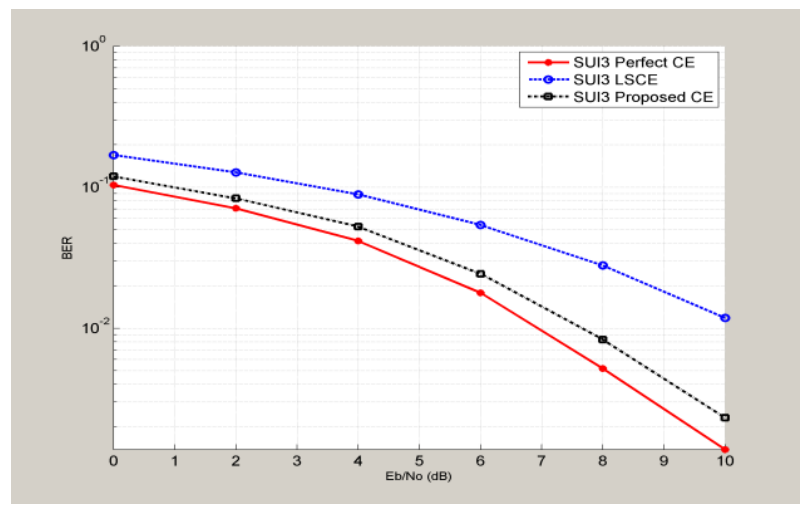

(c) SUI-3 Channel Model

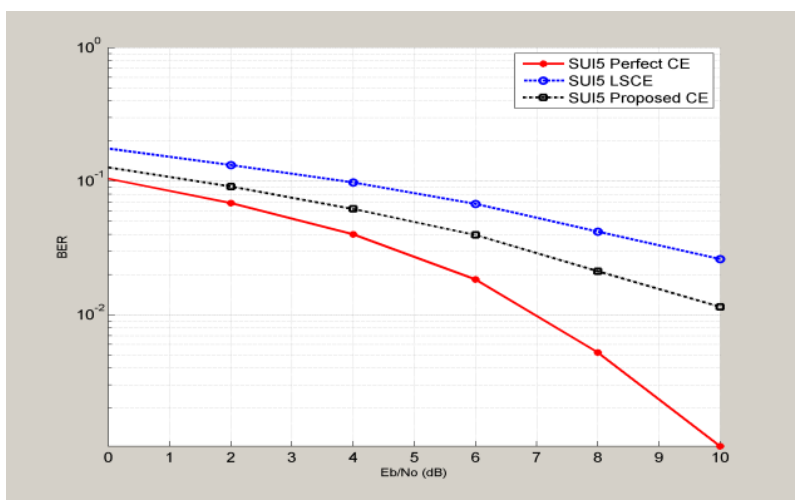

(e) SUI-5 Channel Model the proposed scheme is better than the LS estimator. At high SNR the proposed scheme exhibit slightly faster decay rate than the LS estimator for all channel models. Thus, for lower modulation order the proposed scheme exhibits still better performance.

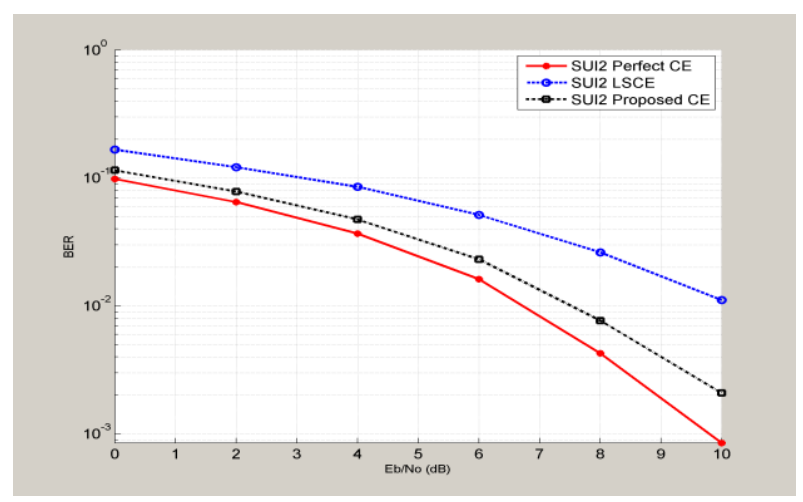

(b) SUI-2 Channel Model

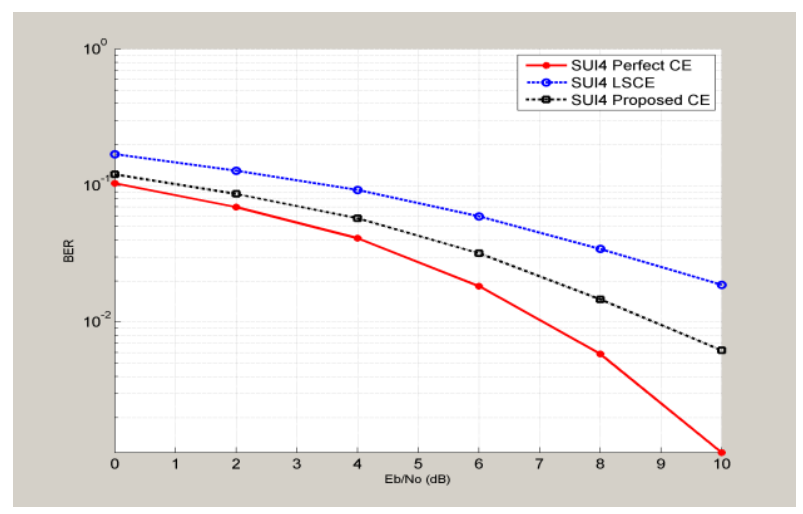

(d) SUI-4 Channel Model

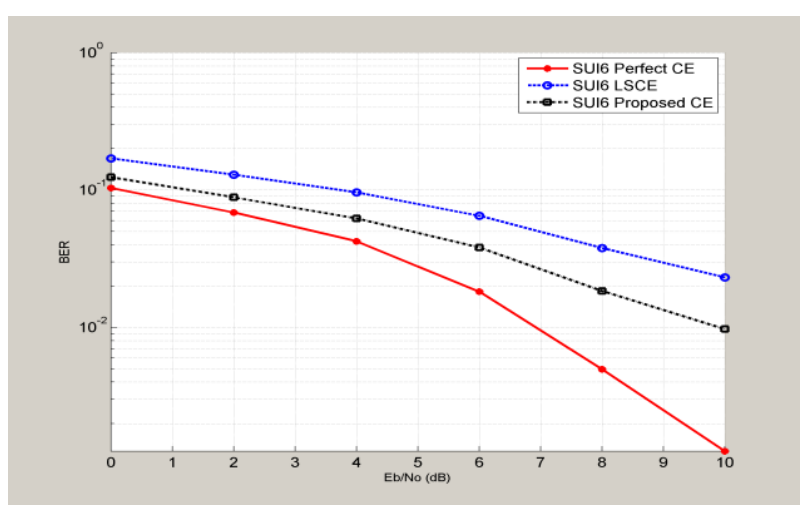

(f) SUI-6 Channel Model

Fig 2: BER Performance of 2X4 SFBC MIMO-OFDM System with different channel models, 64-QAM Modulation.

Table 2. BER values for $\mathrm{LS}$ and proposed Estimator for $\mathrm{Eb} / \mathrm{No}=6 \mathrm{~dB}, 64-\mathrm{QAM}$ Modulation

\begin{tabular}{|l|l|l|l|l|l|l|}
\hline \multicolumn{1}{|c|}{ Channel } & SUI-1 & SUI-2 & SUI-3 & SUI-4 & SUI-5 & SUI-6 \\
\cline { 1 - 6 } Estimator & & & & & & \\
\hline LS CE & 0.04771 & 0.05146 & 0.05396 & 0.05949 & 0.06774 & 0.06497 \\
\hline
\end{tabular}




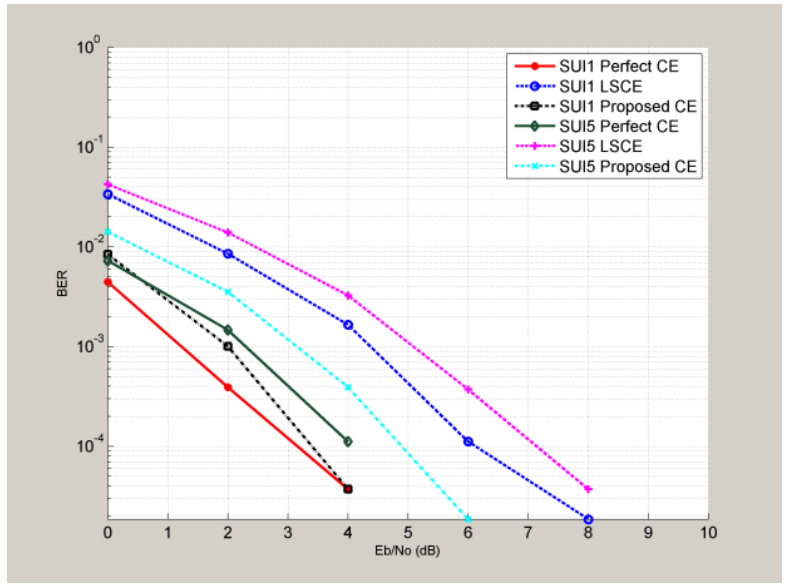

Fig 3: BER Performance of 2X4 SFBC MIMO-OFDM System with SUI-1 and SUI-5 channel model, QPSK Modulation.

\section{CONCLUSIONS}

A combined time and frequency domain approach for channel estimation for SFBC MIMO-OFDM system is proposed. The BER performance of proposed scheme is tested under different SUI channel models and compared with the pilot assisted LS estimator. The proposed scheme exhibits better performance for the channel with low delay spread. The proposed scheme exhibits low decay rate especially when we use higher order modulation scheme and channel with large delay spread. Future work will include performance testing and improvement of the estimator under high mobility channel conditions. Work on reduction in computational complexity of the estimator can also be undertaken.

\section{REFERENCES}

[1] S. M. Alamouti, "A simple transmit diversity technique for wireless communications," IEEE Trans. Sel. Areas Commun., vol. 16, pp. 1451-1458, Oct. 1998.

[2] V. Tarokh, H. Jafarkhani, and A. R. Chalderbank, "Space time block codes from orthogonal design," IEEE Trans. Inf. Theory, vol. 45, no. 5, pp. 1456-1467, July 1999.

[3] V. Tarokh, H. Jafarkhani, and A. R. Chalderbank, "Space-time block coding for wireless communications: performance results," IEEE Trans. Sel. Areas Commun., vol. 17, no. 3, pp. 451-460, March 1999.

[4] G. G. Raleigh and J.M. Cioffi, "Spatio-temporal coding for wireless communications," IEEE Trans Commun., vol. 46, pp. 357-366, Mar. 1998.

[5] H. Bölcskei, D. Gesbert, and AJ. Paulraj, "On the capacity of OFDM-based spatial multiplexing systems," IEEE Trans. on Commun., vol. 50, pp. 225-234, Feb. 2002.

[6] D. Agarwal, V. Tarokh, A. Naguib, and N. Seshadri, "Space-time coded OFDM for high data rate wireless communication over wideband channels," in Proc. IEEE VTC, vol. 3, 1998, pp. 2232-2236.

[7] A. J. Paulraj, D. A. Gore, R. U. Nabar, and H. Boelcskei, "An overview of MIMO communications - A key to gigabit wireless," Proc. IEEE, vol. 92, no. 2, pp. 198218, Feb. 2004.

[8] H. Bölcskei and A. J. Paulraj, "Space-frequency coded broadband OFDM systems," in Proc. IEEE WCNC, vol.

\section{1, 2000, pp. 1-6.}

[9] H. Bölcskei and A. J. Paulraj, "Space-frequency codes for broadband fading channels," in Proc. IEEE ISIT, 2001, p. 219.

[10] H. Bölcskei, M. Borgmann, and A. J. Paulraj, "Spacefrequency coded MIMO-OFDM with variable multiplexing-diversity tradeoff," in Proc. IEEE ICC, vol. 4, 2003, pp. 2837-2841.

[11] A. F. Molisch, M. Z. Win, and J. H. Winters, "Spacetime-frequency (STF) coding for MIMO-OFDM systems," IEEE Commun. Lett., vol. 6, pp. 370-372, Sept. 2002.

[12] Jan Mietzner, Robert Schober, Lutz Lampe, Wolfgang H. Gerstacker, Peter A. Hoeher, Multiple-Antenna Techniques for Wireless Communications - A Comprehensive Literature Survey, IEEE communications survey and tutorials, Vol II No 2.pp. 87-105, Second quarter 2009

[13] Mohammed Abuthinien, Sheng Chen, and Lajos Hanzo, Semi-blind Joint Maximum Likelihood Channel Estimation and Data Detection for MIMO Systems, IEEE Signal Processing Letters, pp.202-205, 2008

[14] M. Biguesh and A. B. Gershman, "MIMO channel estimation: optimal training and tradeoffs between estimation techniques," in Proc. of IEEE International Conference on Communications, 2004, vol. 5, pp. 26582662.

[15] A Gorokhov and P Loubaton, "Blind identification of MIMO-FIR systems: A generalized linear prediction approach,” Signal Processing, vol. 73 (1-2), pp. 105-124, 1999.

[16] A. Medles and D. T. M. Slock, "Linear precoding for spatial multiplexing MIMO systems: blind channel estimation aspects," in Proc. Of IEEE International Conference on Communications, 2002, vol. 1, pp. 401405 .

[17] Ding Zhi and Qiu Li, "Blind MIMO channel identification from second order statistics using rank deficient channel convolution matrix," IEEE Trans. on Signal Processing, vol. 51, no. 2, pp. 535-544, 2003. 
[18] A. Medles, D. T. M. Slock, and E. De Carvalho, "Linear prediction based semi-blind estimation of MIMO FIR channels," in Proc. of IEEE Third Workshop on Signal Processing Advances in Wireless Communications, 2001, pp. 58-61.

[19] A. Medles and D. T. M. Slock, "Semiblind channel estimation for MIMO spatial multiplexing systems," in Proc. of IEEE Vehicular Technology Conference, 2001, vol. 2, pp. 1240-1244.

[20] Y. (G.) Li, N. Seshadri, S. Ariyavisitakul, "Channel Estimation for OFDM Systems with Transmitter Diversity in Mobile Wireless Channels," IEEE J. selec. areas. Commun., vol.17, No.3, March. 1999.

[21] Y. Li, "Simplified Channel Estimation for OFDM Systems with Multiple Transmit Antennas," IEEE Trans. Wireless Comm., vol. 1, 2002.

[22] F. Wan, W.-P. Zhu, and M. N. S. Swamy, "A semiblind channel estimation approach for MIMO-OFDM systems," IEEE Trans. Signal Process.,vol. 56, no. 7, pp. 2821-2834, Jul. 2008.
[23] Mohammad Torabi, Sonia A"issa, and M. Reza Soleymani, "On the BER Performance of SpaceFrequency Block Coded OFDM Systems in Fading MIMO Channels," IEEE Trans. Wireless Comm., vol. 6, No. 4, pp. 1366-1373, 2007.

[24] M. Hsieh and C.Wei, "Channel estimation for OFDM systems based on comb-type pilot arrangement in frequency selective fading channels," IEEE Trans. Consumer Electron., vol. 44, no. 1, Feb. 1998.

[25] Shen, D., Diao, Z., Wong, K. K., \& Li, V. O. K. "Analysis of pilot-assisted channel estimators for OFDM systems with transmit diversity," IEEE Transactions on broadcasting, Vol. 52, No. 2, June 2006.

[26] Roger B. Marks, "The IEEE 802.16 Wireless MAN Standard for Wireless Metropolitan Area Networks", IEEE C802.16-02/09.

[27] V. Erceg, K.V.S. Hari, M.S. Smith, D.S. Baum, "Channel Models for Fixed Wireless Applications", Contribution to IEEE 802.16.3, July 2000. 